\title{
Study of a solar concentrator for space based on a diffractive/refractive optical combination
}

\author{
Céline Michel $^{1}$, Jérôme Loicq ${ }^{1}$, Fabian Languy ${ }^{2}$, Alexandra Mazzoli ${ }^{1}$, \\ Serge Habraken ${ }^{1,2}$ \\ ${ }^{1}$ Centre Spatial de Liège, Liège, Belgium \\ ${ }^{2}$ University of Liège - Hololab, Liège, Belgium \\ celine.michel@doct.ulg.ac.be
}

\begin{abstract}
This paper presents a new design of a planar solar concentrator at $10 \times$ with spectral splitting focusing on two separated PV cells, allowing independent control. Optical elements, blazed diffraction grating and Fresnel lens, are optimized.

(C) 2012 Optical Society of America

OCIS codes: (220.1770) Concentrators, (350.6050) Solar energy, (350.6090) Space optics, (080.4298) Nonimaging optics, (220.3630) Lenses, (050.1950) Diffraction gratings
\end{abstract}

Satellites, more and more present, produce their energy from the sun, by the use of photovoltaic solar panels composed of multi-junction cells (MJ). However, given the current energy needs, the required collector area is increasing. But solar cells represent both the most expensive and heaviest element of the solar generator panel. To reduce the price/Watts, increase the ratio Watts/kg, and increase the solar flux on cells (great advantage for deep space missions), one solution is solar concentration.

\section{Description}

The concentrator in this paper is a combination of:

1. A diffractive element (a blazed diffraction grating [1]) which separates spatially and spectrally the incident solar flux into two parts: the non-diffracted IR on one hand, and the near IR - visible - UV, 1st order diffracted.

2. A refractive element (a flat cylindrical Fresnel lens [2]) which focuses each part of the spectrum on two specific $\mathrm{PV}$ cells. The flat shape of lenses is chosen to make easier the storage of the concentrator during the launch phase. It will be deployed by a vertical pop-up system.

Principle is depicted in Figure 1. Secondary concentrators allow a decreased sensitivity to a tracking error. The diffraction grating - Fresnel lens system is ideally built from silicone (silicone DC93-500, already spatialized [3]). The silicone film will be stretched between the edges of the support, and thus be self-supporting.

Advantages This system presents several advantages due to additional degrees of freedom provided by the spectral separation, in addition to reduce the cost and the mass, increase the incident solar flux on PV cells, and thus their performances too, as well as make easier the fabrication by use of roll-to-roll technology.

Indeed, the use of two single-junction PV cells, spatially separated, instead of the usual MJ cells is attractive. The two main limitations [4] of these cells are (1) the lattice matching condition between the materials at the interfaces between the junctions, and (2) the current matching condition, since the junctions are connected in series. The first limitation restricts the choice of materials, preventing a potentially better combination. In our configuration, the junctions are separated and the choice of materials is open. The second limitation implies that the less effective junctions limits the current of all other junctions, the excess power that could provide other junctions is thus lost. This makes the system more sensitive to a change in the spectrum of incident light with respect to that of the calibration, or to the aging of the junctions. If the cells are spatially separated, the maximum power of each can be recovered, providing in theory, higher performance. Moreover, duplication of surfaces enables a better heat transfer, and therefore a lower cell temperature, so the performance will be less degraded [5] compared to the usual case where all the light is concentrated on a single MJ cell. Furthermore, the spectral separation gives the possibility to use an anti-reflective coating specific to each part of the spectrum incident on the two cells, which is more efficient than using a single coating to cover the full spectrum. 


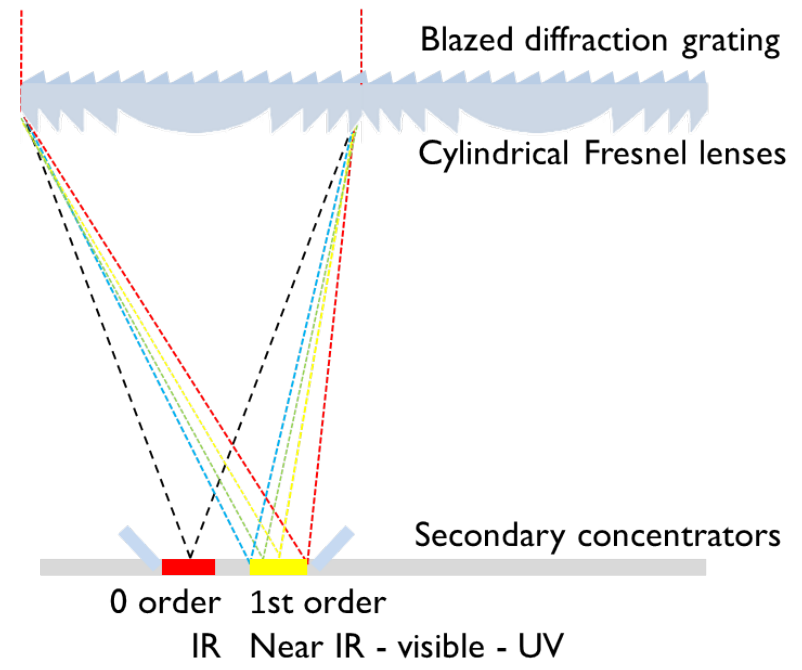

Fig. 1. Solar concentrator design, scale not representative.

In addition, this concentrator is not limited to the space sector, it is fully adaptable for terrestrial applications. In this case, the thermal limitations and tracking are not the same, and thus, a much higher concentration factor could be achieved. To make the project sustainable on Earth, some modifications are needed. We are working on this type of adaptations, such a coupling with a waveguide, etc., the benefits of spectral splitting remaining the same.

\section{System optimization}

In order to obtain the optimal optical configuration, two main optimizations are performed: (1) the maximization of output power, reaching the most efficient system, and (2) the minimization of PV cell size (accordingly focal spots), reducing the surface and thus minimizing the cost and weight. For this purpose, several simulation programs and optimizations have been developed, particularly on the basis of ray tracing:

- First, for the optimization of the shape of the Fresnel lens at any point based on the non-imaging optics, which gives a non-symmetric lens, while the diffraction grating is described by the scalar diffraction theory [6] (since the diffraction period is large enough).

- Second, to calculate the dimensions of focal spots according to the parameters of the diffraction grating and the Fresnel lens.

- Finally, to obtain the spatial and spectral distribution of irradiance in the focal plane, including transmission and optical efficiencies.

\section{Present performance}

An optimal configuration has been established based on a compromise between maximizing the total photocurrent, minimizing dimensions (maximizing geometric concentration factor) and maximizing the transmission efficiency of optical elements. The results are promising, mainly with an overall optical efficiency of about $75 \%$, an electrical power density of $266.5 \mathrm{~W} / \mathrm{m}^{2}$, an output power equal to $87 \%$ of that provided by a planar PV panel covered with classic MJ cells under optimal conditions, and geometrical factors of concentration of about $10 \times$ for each cell. So, this approach would allow an economy of about $85 \%$ of PV cells. A view of the spatial and spectral distribution of light on cells is included in Figure 2. We observe the spatial separation of the two focal spots and a spectral distribution, demonstrating the feasibility of the concept, taking into account the realistic disc size of the sun. Note that the spectral overlap induces no large losses since the IR cell is still able to convert photons of wavelength below $900 \mathrm{~nm}$, although this is not the spectral range where it is most effective. 


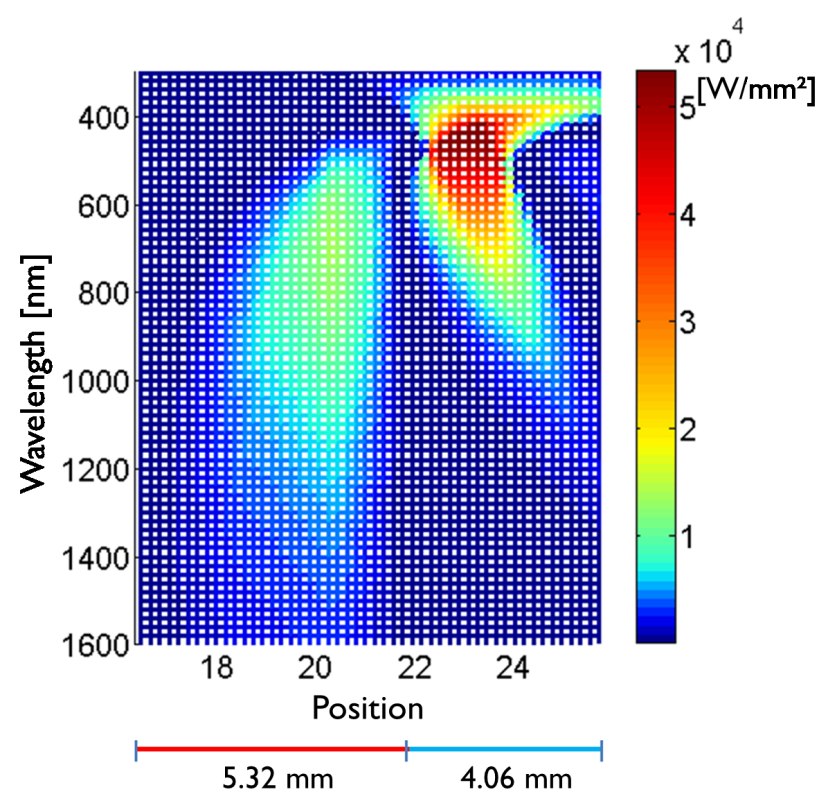

Fig. 2. Power density $\left[\mathrm{W} / \mathrm{mm}^{2}\right]$ reaching cells, according to the wavelength and the position on the cell. The two focal spots are well spatially separated, with a visible spectral separation.

\section{Conclusions}

Numerical results, including the optical efficiency, show that the concept is realistic and that the inevitable optical losses due to the concentration and diffraction are acceptable and partly recovered by the degree of freedom offered by the spatial separation of PV junctions. The performed work has thus demonstrated the viability of a new solar concentrator design, avoiding the limitations of MJ cells. Performance is promising, studies of sensitivity to misalignment, as well as adding of secondary concentrators will complete the study. In the future, thermal design of a space solar panel with the described concentrator will be presented. Adaptations to a terrestrial concentrator is under progress and will be published soon.

\section{References}

1. Eugene Hecht, Optics, Pearson Education, Inc., p 478, 2002

2. R. Leutz, A. Suzuki, A. Akisava \& T. Kashiwagi, Developments and designs of solar engineering Fresnel lenses, Proceedings Symposium on Energy Engineering (SEE 2000), vol. 2, p 759-765, 2000

3. Joyce A. Dever, Bruce A. Banks \& Li Yan, Vacuum ultraviolet radiation effects on DC93-500 silicone film, Space Technology Proceedings, vol. 6, p 123-140, 2006

4. H. Cotal, C. Fetzer, J. Boisvert, G. Kinsey, R. King, P. Hebert, H. Yoon \& N. Karam, III-V multijunction solar cells for concentrating photovoltaics, Energy \& Environnemental Science, n 2, p 174-192, 2009

5. K. Nishioka, T. Takamoto, T. Agui, M. Kaneiwa, Y. Uraoka \& T. Fuyuki, Evaluation of temperature characteristic of high-efficiency InGaP/InGaAs/Ge triple-junction solar cells under concentration, Solar Energy Materials \& Solar Cells, n 85, p 429-436, 2005

6. Joseph W. Goodman, Introduction to Fourier Optics, The McGraw-Hill Companies, Inc., chapter 3, 1968 